\title{
Are the causes of obesity primarily environmental? No
}

Timothy M Frayling, the author of this Head to Head article, has alerted us to an error in the penultimate paragraph ( $B M J$ 2012;345:e5844, doi:10.1136/bmj.e5844). The sixth sentence in this paragraph stated that "inactivity in children preceded increases in percentage body fat, but increased body fat percentage did not precede reduced physical activity." This is incorrect and should have read: "Inactivity in children did not precede increases in percentage body fat, but increased body fat percentage did precede reduced physical activity."

Cite this as: BMJ 2012;345:e6217

๑ BMJ Publishing Group Ltd 2012 